\title{
Episodio de cizalladura del 10-9-2017 en el aeropuerto de Barcelona
}

\author{
Alejandro Méndez Frades ${ }^{1}$ (amendezf@aemet.es) \\ María Rosa Pons Reynés ${ }^{2}$ (mponsr@aemet.es) \\ Inés Santos Atienza ${ }^{3}$ (isantosa@aemet.es) \\ Sergi González Herrero ${ }^{4,5}$ (sgonzalezh@aemet.es) \\ Ramón Pascual Berghaenel ${ }^{4}$ (rpascualb@aemet.es) \\ Agustí Pladevall Boix ${ }^{4}$ (apladevallb@aemet.es) \\ Alfons Callado Pallarés ${ }^{4}$ (acalladop@aemet.es) \\ Marc Compte Rovirola ${ }^{4}$ (mcompter@aemet.es) \\ Juan Pablo Simarro Grande ${ }^{6}$ (jsimarrog@aemet.es)
}

${ }^{1}$ AEMET / Dirección de Planificación, Estrategia y Desarrollo Comercial / Unidad de Meteorología Aeronáutica ${ }^{2}$ AEMET / Delegación Territorial en Cantabria

${ }^{3}$ AEMET / Dirección de Producción e Infraestructuras / Área de Aplicaciones

${ }^{4}$ AEMET / Delegación Territorial en Cataluña

${ }^{5}$ Universitat de Barcelona / Departamento de Física Aplicada - Meteorología

${ }^{6}$ AEMET / Delegación Territorial en la Comunidad Valenciana

\begin{abstract}
RESUMEN
El estudio de este episodio se realiza a solicitud del gestor de navegación aérea ENAIRE para analizar en profundidad una situación operativa crítica que tuvo lugar en el aeropuerto internacional de Barcelona-El Prat el pasado 10 de septiembre de 2017. Desde el punto de vista meteorológico, el elemento más significativo de este episodio fue la cizalladura horizontal del viento presente en el ámbito del aeropuerto que, debido a que este estaba al máximo de frecuencia temporal de operaciones, tuvo un gran impacto en la operatividad del centro de control de área.
\end{abstract}

PALABRAS CLAVE: aeronáutica; cizalladura; impacto; corriente de densidad atrapada en la costa; ensemble.

\section{OBJETIVO}

Caracterización de la situación meteorológica de dicho episodio, analizando todas las observaciones disponibles y las salidas de modelos números de predicción operativos, además de evaluar el valor añadido que podrían aportar las integraciones del modelo HARMONIE AROME utilizando una resolución horizontal de $1 \mathrm{~km}$ así como el ensemble de corto plazo AEMET- $\gamma$ SREPS que se encuentra en fase preoperativa.

\section{ANÁLISIS}

En la figuras 1 y 2, se muestra el análisis del entorno sinóptico a partir del modelo ECMWF para el día 10-9-2017 a las 12 UTC. En niveles medios y altos (figura 1) se observa el paso de una vaguada con un núcleo frío que alcanza los $-22{ }^{\circ} \mathrm{C}$ conducida por un máximo de viento trasero de $100 \mathrm{kt}$ y en su parte delantera de $80 \mathrm{kt}$, lo que posibilitó su desplazamiento progresivo hacia el E. 

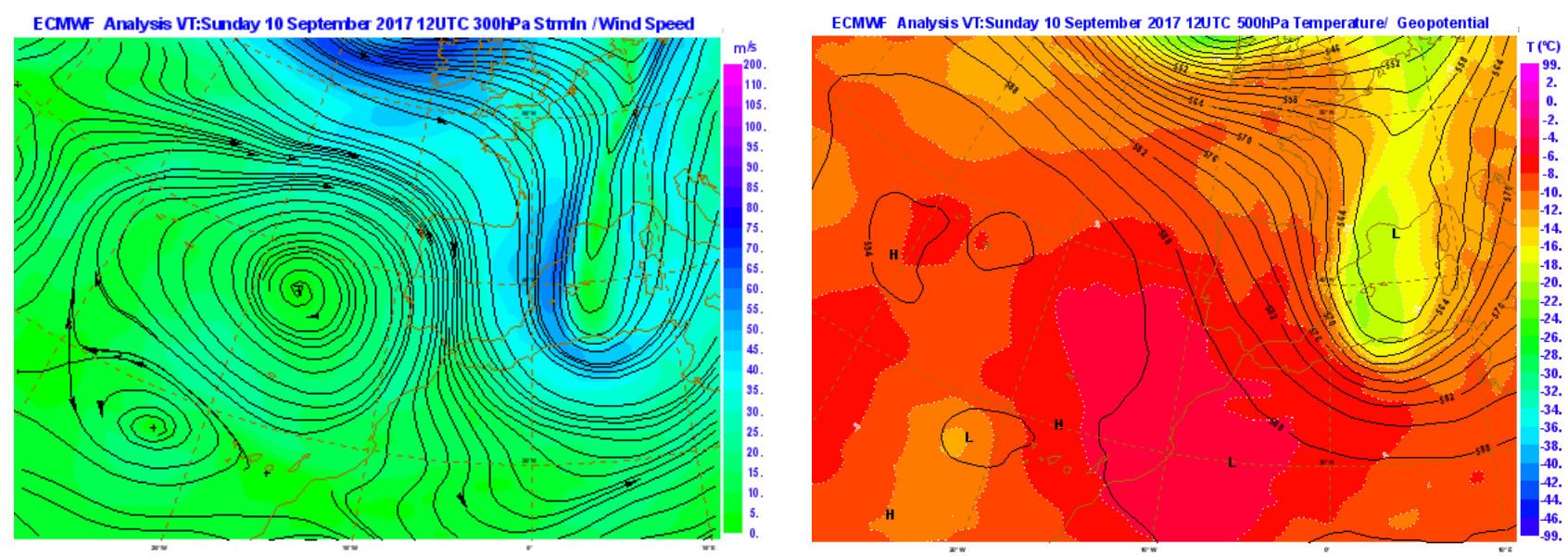

Figura 1. Análisis del modelo ECMWF para el día 10-9-2017 a las 12 UTC. A la izquierda, se representa el viento en $300 \mathrm{hPa}$. A la derecha, el geopotencial y la temperatura en $500 \mathrm{hPa}$.

Fuente: Centro Europeo de Predicción a Medio Plazo (ECMWF).

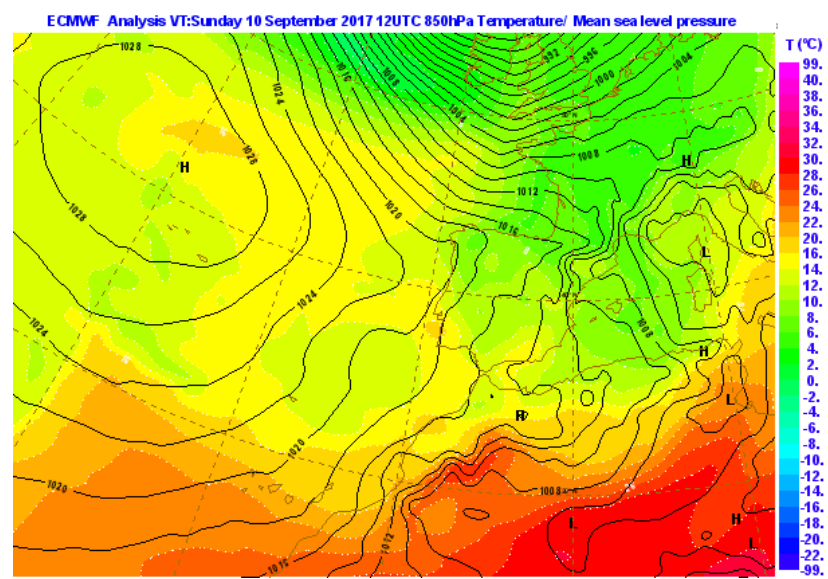

En el análisis de superficie (figura 2) se identifica un extenso anticiclón atlántico (centrado al NW de las islas Azores) y un centro de baja presión localizado sobre Liguria. La localización de ambos centros de acción sinópticos genera un flujo de componente NW sobre la Península. Este flujo, tras interaccionar con los obstáculos orográficos de la Península, da lugar a una configuración del campo isobárico en forma de dipolo orográfico, i.e. una mesoalta a barlovento y una mesobaja a sotavento.

Figura 2. Análisis del modelo ECMWF para el día

10-9-2017 a las 12 UTC. Se representa la temperatura en $850 \mathrm{hPa}$ y la presión a nivel medio del mar. Fuente: Centro Europeo de Predicción a Medio Plazo (ECMWF).

\section{DIAGNOSIS}

\subsection{Caracterización del entorno mesoescalar}

La caracterización mesoescalar consistirá en dar un valor añadido al campo de viento en superficie registrado en un conjunto de puntos discretos en el espacio y en un instante determinado por las estaciones automáticas de AEMET. De esta forma, se identificarán los flujos que se establecen como consecuencia de la deformación del campo isobárico (figuras 3 y 4).

En particular, de la figura 3 destacan las siguientes estructuras:

- Viento del W-NW soplando sobre el valle del Ebro, afectando a la mitad sur de Cataluña, incluyendo el Pla de Lleida, toda la provincia de Tarragona y el interior y extremo sur de la de Barcelona.

- Viento del $\mathbf{N}$ en el Empordà, que es la aceleración que sufre la Tramontana por producirse en el extremo de la cordillera pirenaica. 


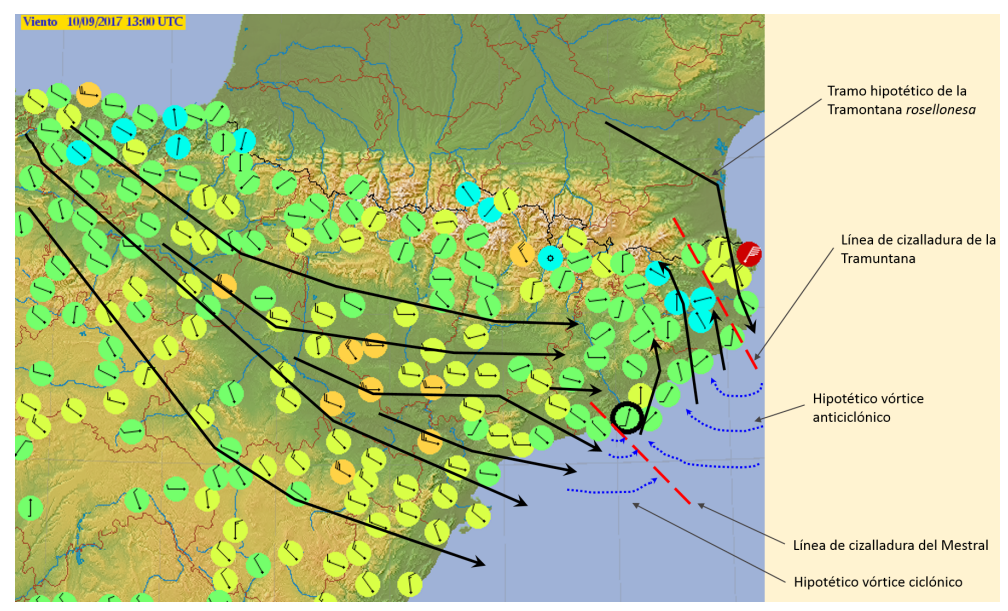

Figura 3.

Caracterización del entorno mesoescalar

del día 10-9-2017 a las 13 UTC.

- Viento del S en el litoral de Barcelona y parte de Girona. Este flujo es habitual cuando sopla el Mestral Tramontana, el cual se genera por la curvatura anticiclónica de la Tramontana y/o curvatura ciclónica del Mestral.

En las horas posteriores (figura 4) se puede observar cómo la línea de cizalladura del Mestral cambia de posición, habida cuenta de los vientos registrados en el aeropuerto: viento del NW a las 16 UTC y del NE a las 17 UTC.
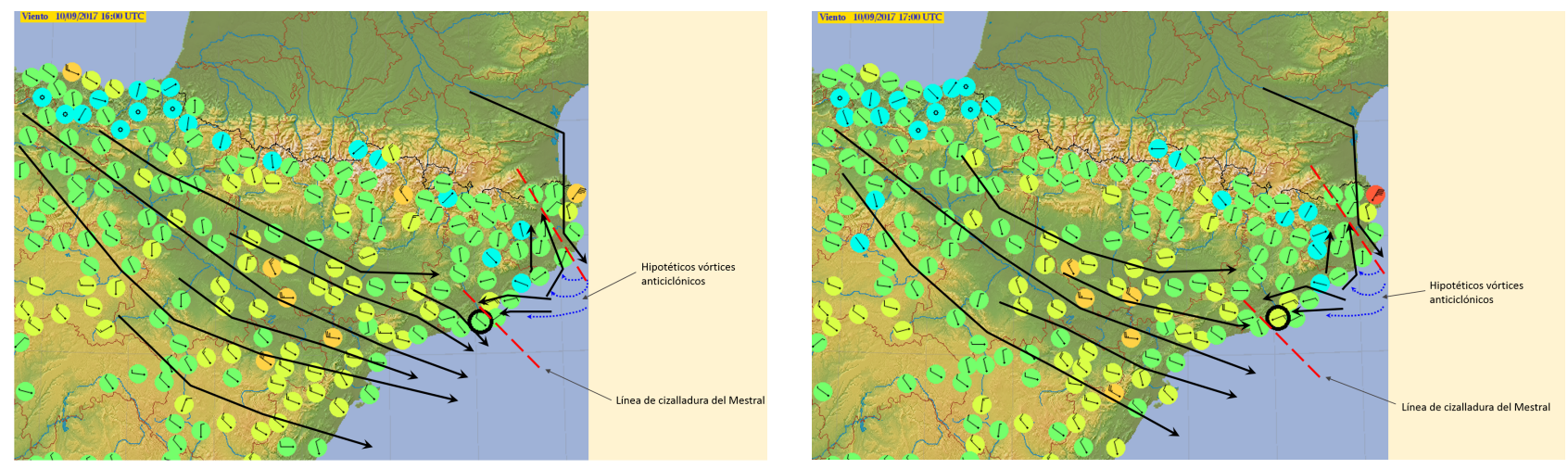

Figura 4. Caracterización del entorno mesoescalar del día 10-9-2017 en las horas posteriores: a las 16 UTC (izquierda) y a las 17 UTC (derecha).

\subsection{Caracterización del entorno micrometeorológico}

El conocimiento del viento en superficie en cada uno de los sensores localizados en las cabeceras de las pistas del aeropuerto constituye una valiosa fuente de información ya que, unido al análisis mesoescalar precedente, permite hacer un seguimiento espaciotemporal casi continuo e incluso inferir la situación micrometeorológica embebida en este episodio.

En la figura 5 se presenta la secuencia temporal, en intervalos de diez minutos, del viento en superficie observado en cada una de las cabeceras del aeropuerto el día 10-9-2017. En un primer instante (16:10 UTC), aparecía un campo de viento uniforme del NW que se prolongó durante diez minutos. A lo largo de ese intervalo, la configuración del aeropuerto era tal que las aeronaves aterrizaban satisfactoriamente por la cabecera $25 \mathrm{R}$ ya que, para que dicha operación pueda ser culminada con éxito, se precisa de un viento en cara o incluso un viento en cola no superior a $9 \mathrm{kt}$.

A las 16:20 UTC, se mide por primera vez viento del N sobre las cabeceras de arribada. Este hecho ocasionó 5 aterrizajes frustrados a las 16:25 UTC, lo que generó un cambio de configuración de pista, llegando incluso 


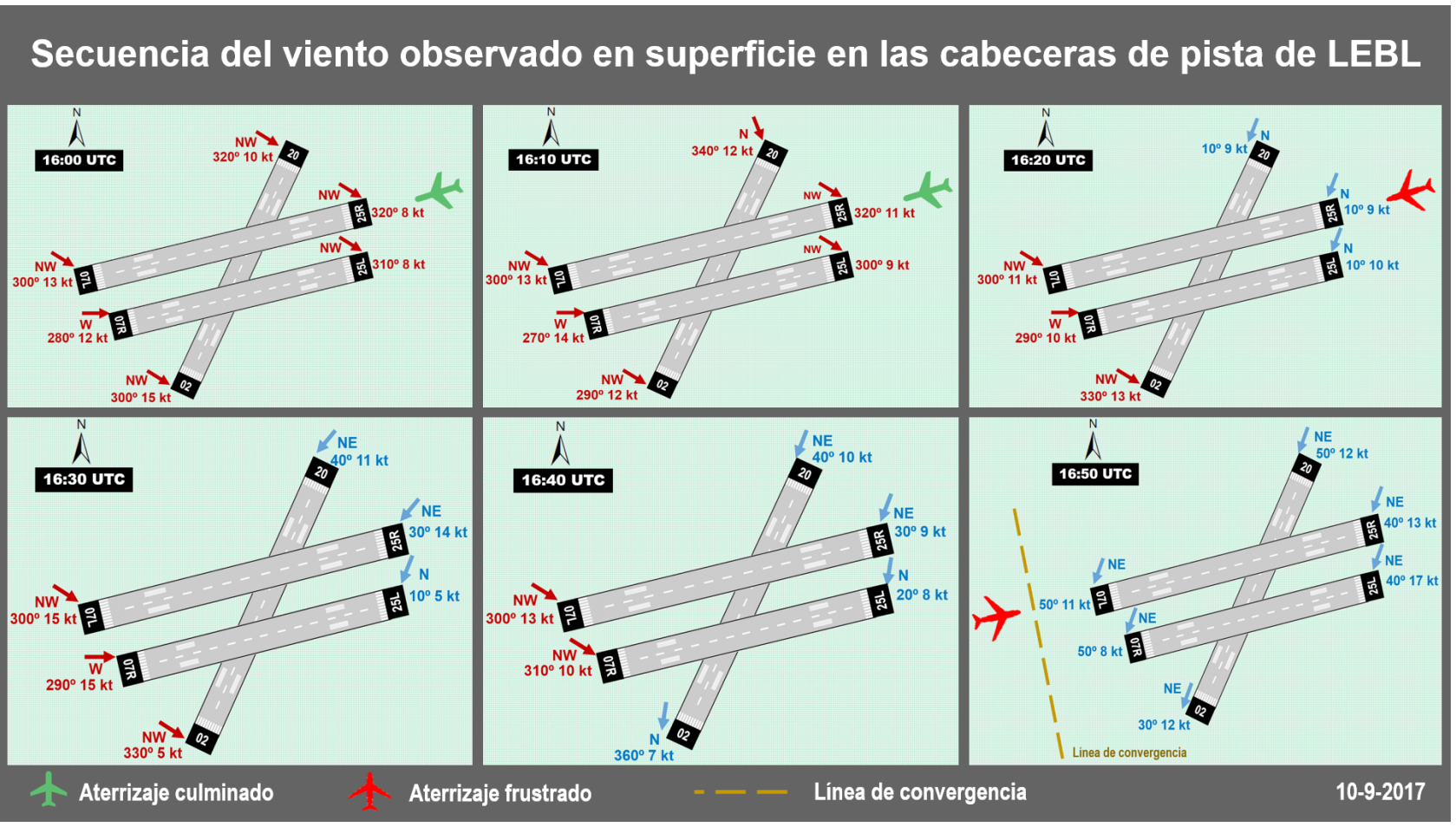

Figura 5. De izquierda a derecha y de arriba hacia abajo, secuencia de viento observado en superficie en cada una de las cabeceras de las pistas del aeropuerto de Barcelona el 10-9-2017 a las horas especificadas:

desde las 16:10 UTC hasta las 16:50 UTC, en intervalos de 10 minutos. Los colores diferencian las direcciones del viento observado en cada una de las cabeceras y las aeronaves que logran aterrizar (negro) y que frustran (gris). Fuente: datos proporcionados por la oficina meteorológica del aeropuerto de Barcelona.

a utilizar la pista transversal, con la consiguiente reducción a la mitad de la capacidad. Es interesante destacar que, a pesar de registrarse el mismo viento en todas las cabeceras de pista a las 16:50 UTC, las aeronaves no podían aterrizar por la cabecera $07 \mathrm{~L}$.

De todo lo anterior, se puede concluir que, entre las 16:10 UTC y las 16:50 UTC, una línea de convergencia —denotada por el cambio del viento horizontal de NW a NE-barrió paulatinamente las pistas del aeropuerto hasta detenerse en las cercanías de este. Dicho emplazamiento imposibilitó a las aeronaves aterrizar, a pesar de que los sensores de viento de todas las cabeceras indicaban un viento en superficie del NE.

\subsection{Modelo conceptual}

El conjunto de procesos físicos precursores del entorno de cizalladura que se está estudiando en el presente trabajo quedan sintetizados con el modelo conceptual de corriente de densidad atrapada en la costa (GoNZÁLEZ et al., 2018).

La incursión del Cierzo-Mestral (cálido y seco) y de la Tramontana (húmeda y fría) en el Mediterráneo, estimula la formación de una línea de convergencia que progresivamente avanza hacia el lado cálido debido a la diferencia de densidad. A lo largo de ese proceso de barrido, la masa más cálida y seca se sitúa por encima de la más húmeda y fría, dando lugar a un cambio súbito en la dirección e intensidad del viento, que rola del NW-SW al NE, lo que supone un entorno favorable a cizalladura horizontal y vertical donde se localiza el aeropuerto (figura 6).

La figura 7 es una representación idealizada del impacto del viento en la vertical durante la operación de aterrizaje de las aeronaves cuando se aproximan al aeropuerto. Así, antes de las 16 UTC (figura 7, parte superior), las aeronaves pueden alcanzar la cabecera de arribada $25 \mathrm{R}$, mientras que en instantes posteriores 


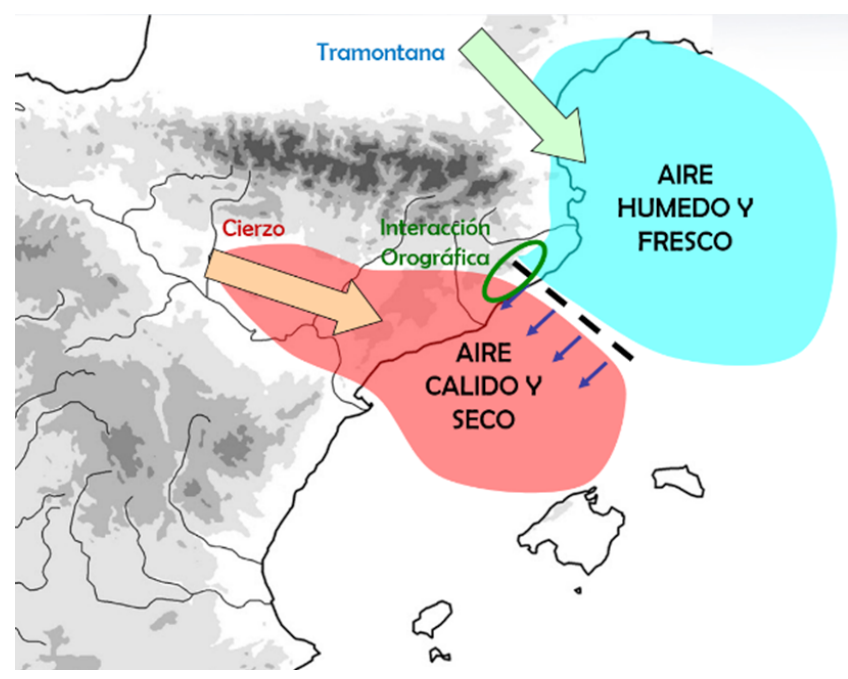

Figura 6.

Esquema conceptual de una corriente de densidad atrapada en la costa de Barcelona.

Los colores identifican la naturaleza térmica de las masas de aire involucradas.

El avance de la masa de aire húmeda y fría sobre la más seca y cálida es precursora de un entorno favorable a cizalladura horizontal sobre el litoral de Barcelona.

Fuente: GonZÁLEZ et al., 2014.

(figura 7, parte inferior), las aeronaves frustran su aterrizaje. Durante esas horas, aunque se observa un viento del NE uniforme sobre las pistas, las aeronaves en aproximación sienten, por encima de unos $1000 \mathrm{ft}$ o a veces menos, viento de cola que les afecta a su sustentación. Por este motivo, cuando alcanzan la altura de decisión, se ven obligadas a volver al aire y recorrer un circuito de espera hasta efectuar un nuevo intento de toma de tierra por la cabecera $07 \mathrm{~L}$.
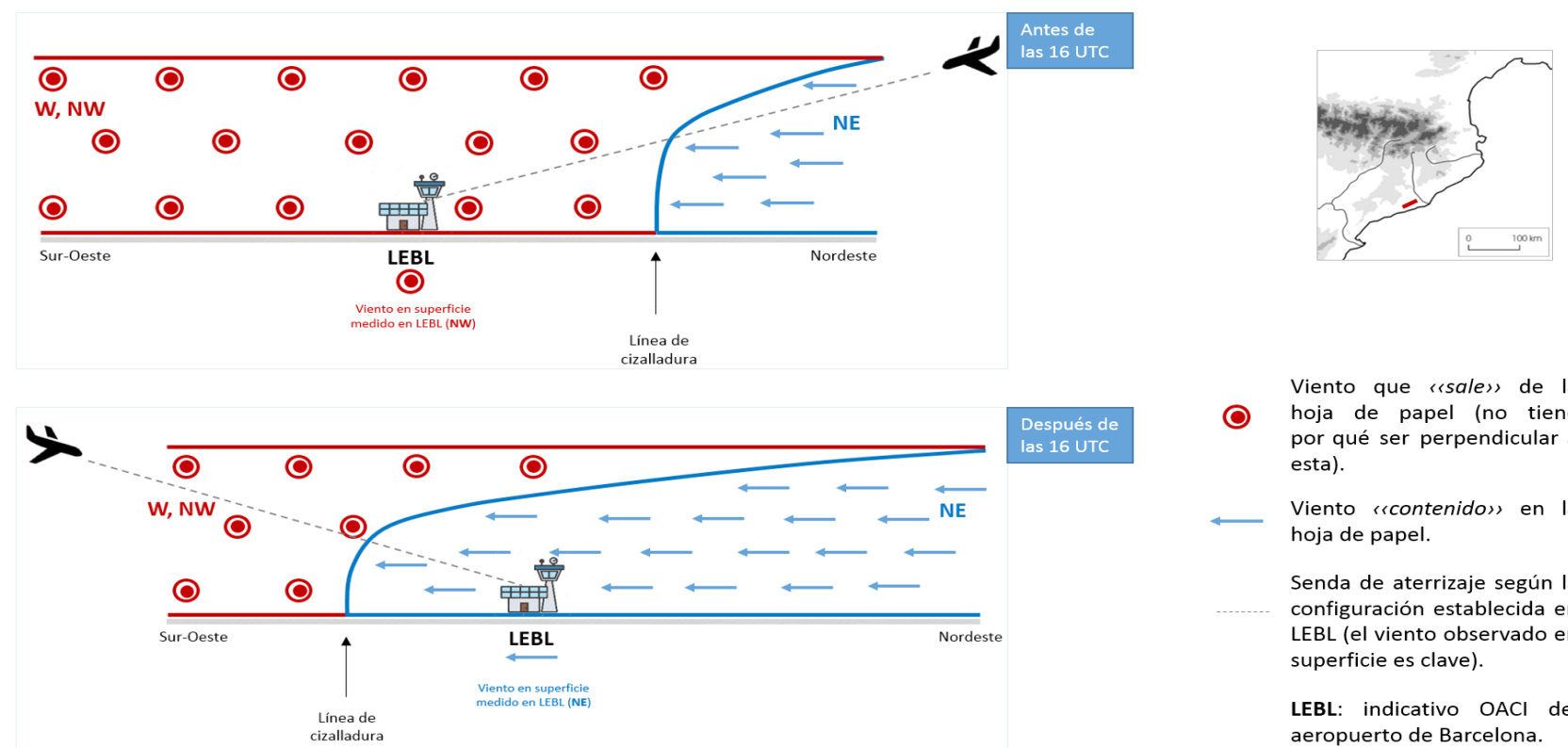

Figura 7. Modelo conceptual de la sección vertical del viento en las inmediaciones del aeropuerto y su impacto en la aproximación y aterrizaje de las aeronaves sobre el eje de la pista 25R-07L.

Los colores diferencian los dos tipos de viento observados.

\section{PREDICCIÓN}

\subsection{Modelo determinista HARMONIE AROME $2,5 \mathrm{~km}$}

El modelo HARMONIE AROME operativo en AEMET está desarrollado por los consorcios ALADIN y HIRLAM. Se trata de un modelo de predicción determinista, de área limitada, no hidrostático con un núcleo dinámico basado en una discretización semilagrangiana y semiimplícita, cuya resolución horizontal es de 2,5 km. Cada 3 horas se realizan predicciones cuya validez llega a H+48 (Bengtsson et al., 2017). En este caso se evaluará el viento pronosticado a 10 metros para el día 10-9-2017 en la pasada de las 6 UTC. 
En la figura 8 se presenta el viento pronosticado a 10 metros para el día 10-9-2017 en la pasada de las 6 UTC para los alcances H+8 (en Cataluña) y H+10 (en Barcelona). En particular, el viento previsto para $\mathrm{H}+8$ está en consonancia con la diagnosis mesoescalar precedente, en la que las observaciones señalaban un viento del S-SW sobre el aeropuerto (figura 8).
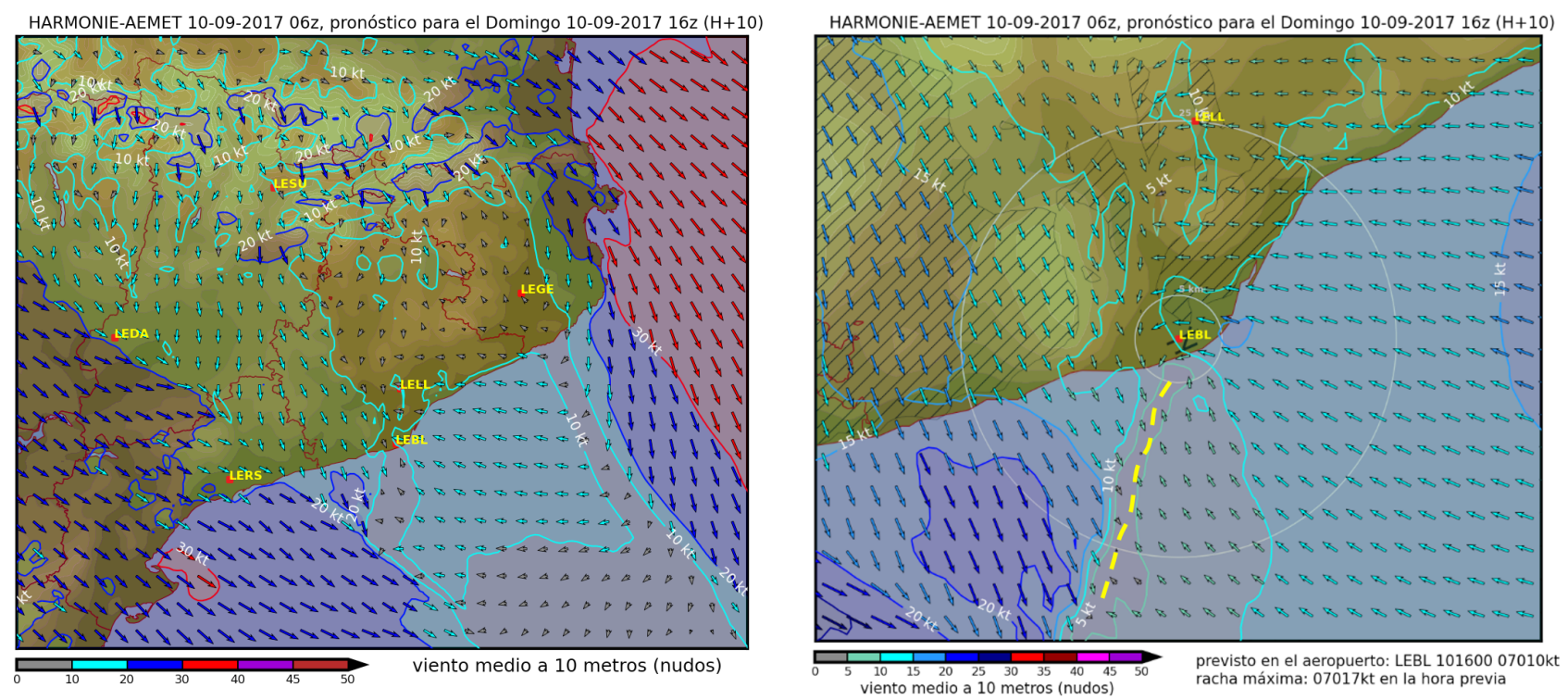

Figura 8. Viento a 10 metros pronosticado por el modelo determinista HARMONIE AROME en el alcance H+10 (Cataluña) y H+10 (Barcelona), respectivamente. Destaca la habilidad que tuvo el modelo en pronosticar la línea de convergencia, la cual se localiza en la discontinuidad horizontal del viento.

En los alcances posteriores, el modelo HARMONIEAROME sí supo ver la línea de convergencia, situándola en las inmediaciones del aeropuerto, la cual marca la discontinuidad en el viento horizontal. Ahora bien, el modelo pronosticó un viento más del E (que no se produjo) que del NE (que sí fue el observado).

En la figura 9 se muestra la sección vertical del viento horizontal y de la energía cinética turbulenta pronosticado por HARMONIE AROME a las 6 UTC del día 10-9-2017 para los alcances H+9 y H+12 en las inmediaciones del aeropuerto. Este campo permite identificar las dos masas de aire involucradas en este episodio lo que confirma el modelo conceptual descrito en el apartado 3.3. En efecto, a la izquierda se reconoce una masa de aire que, entre $1000 \mathrm{hPa}$ y $700 \mathrm{hPa}$, es empujada por un viento del NW (a partir de este nivel se hace prácticamente del N) mientras que, a la derecha, aparece la otra masa de aire implicada, empujada por un viento del SE entre los niveles $1000 \mathrm{hPa}$ y $900 \mathrm{hPa}$. En el alcance posterior se observa el avance de la masa fría y húmeda. La señal de energía turbulenta está relacionada con el grado de inestabilidad estática asociada a la masa de aire cálida y seca.
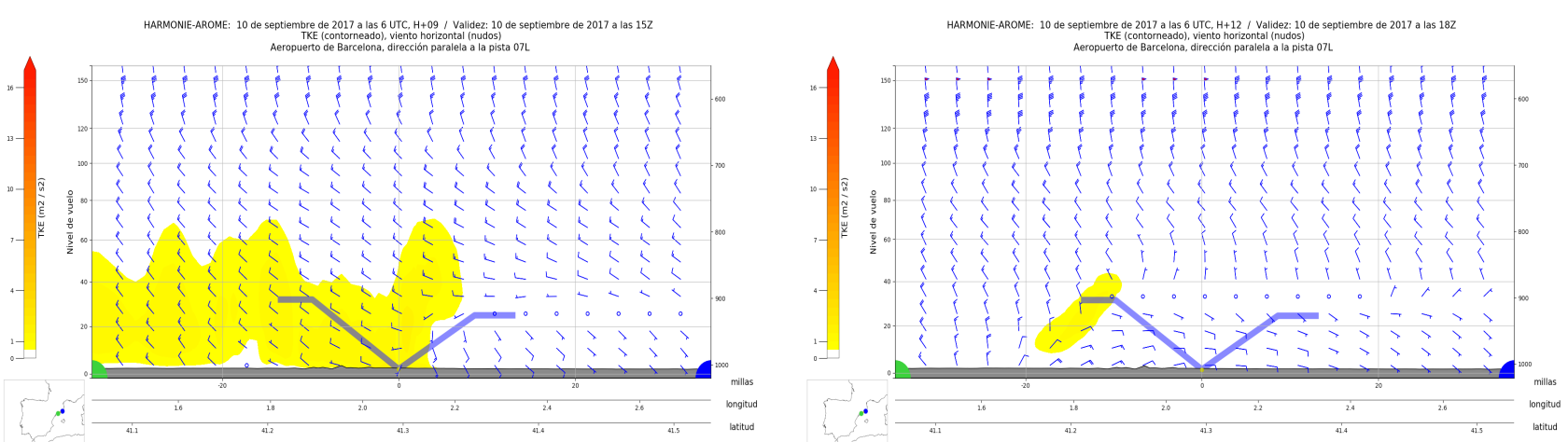

Figura 9. Perfil vertical de la energía cinética turbulenta (amarillo) y del viento horizontal (azul) pronosticado por el modelo HARMONIE AROME en los alcances H+9 (izquierda) y H+12 (derecha), respectivamente.

La línea gruesa en forma de « $\mathrm{V} »$ denota la senda de aproximación. 


\subsection{Modelo de predicción determinista HARMONIE-AROME a 1 km}

En la actualidad, el modelo HARMONIE AROME a 1 km de resolución espacial está siendo utilizado en AEMET para dar apoyo meteorológico y oceanográfico a Puertos del Estado dentro del proyecto SAMOA.

La figura 10 presenta el campo de viento en superficie previsto para el 10-9-2017 en dos alcances concretos (16 UTC y 18 UTC, respectivamente). En ambos, se reconoce plenamente el sistema regional de vientos: Cierzo-Mestral-Tramontana y la línea de convergencia mesoescalar. Ahora bien, cabe destacar la incertidumbre en el posicionamiento de la línea de convergencia en las inmediaciones del aeropuerto a lo largo de los distintos alcances.
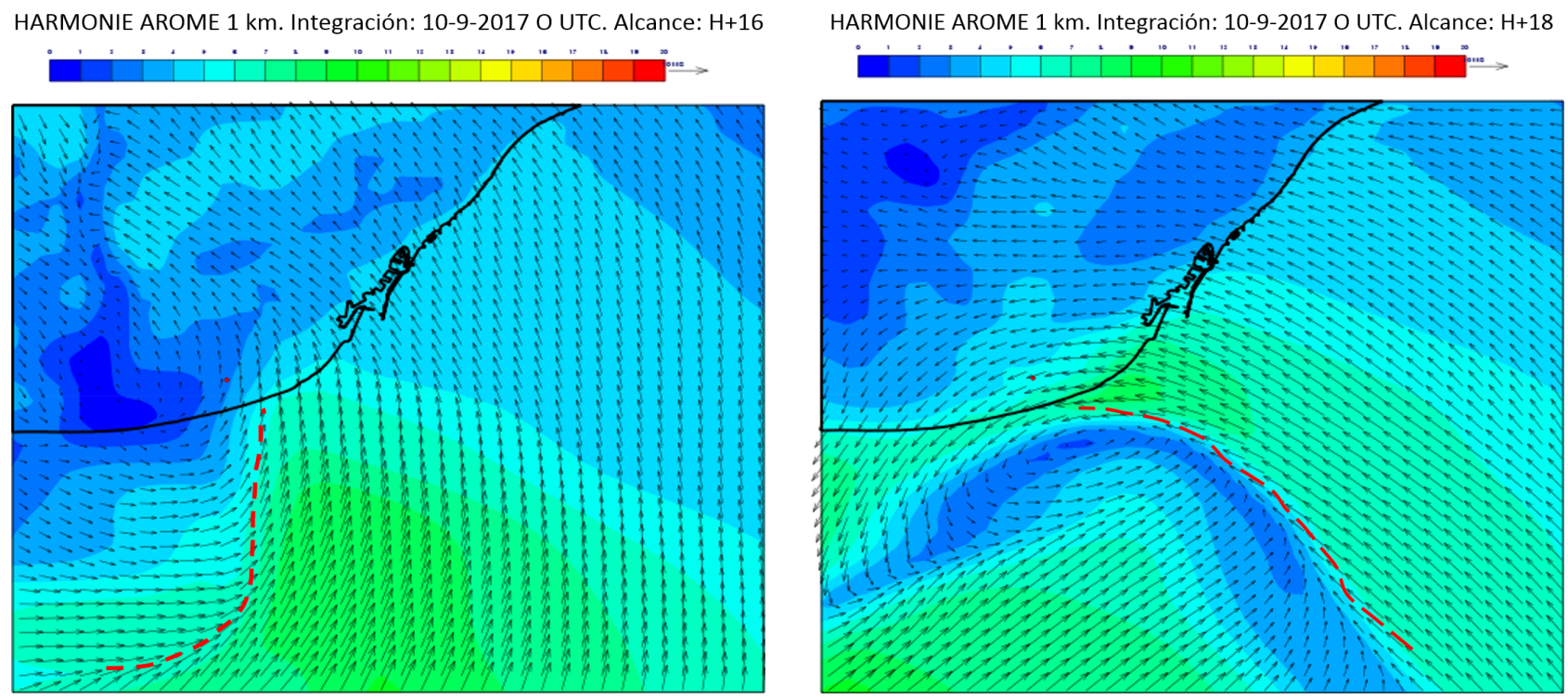

Figura 10. Viento previsto en superficie por el modelo HARMONIE AROME a $1 \mathrm{~km}$ de resolución horizontal, en la pasada de las 0 UTC del 10-9-2017 y alcances H+16 y H+18. La línea discontinua roja representa la línea de convergencia. El punto marcado representa el aeropuerto.

\subsection{Modelo de predicción probabilista AEMET- $\gamma$ SREPS}

Las ecuaciones fundamentales que describen el comportamiento de la atmósfera son altamente no lineales, presentando un comportamiento típico de un sistema caótico, motivo por el cual, es inviable su resolución analíticamente. Por este motivo, el estado del arte de la predicción no posibilita el pronóstico determinista de la línea de cizalladura en el entorno del aeropuerto. Al tratarse de un fenómeno localizado en un entorno muy local, es necesario recurrir a una interpretación probabilista mediante el uso de un sistema de predicción por conjuntos (en inglés, ensemble prediction system, EPS) de corto plazo.

El valor añadido que facilita una visión probabilista podría resumirse de la siguiente manera:

- Hay incertidumbres espaciales y temporales asociadas a la línea de convergencia, reflejadas en el AEMET- $\gamma$ SREPS. La incertidumbre espacial queda bien reflejada en los dos miembros (2 y 3 ) que se muestran en la figura 11.

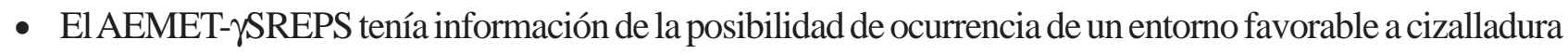
en el aeropuerto. 


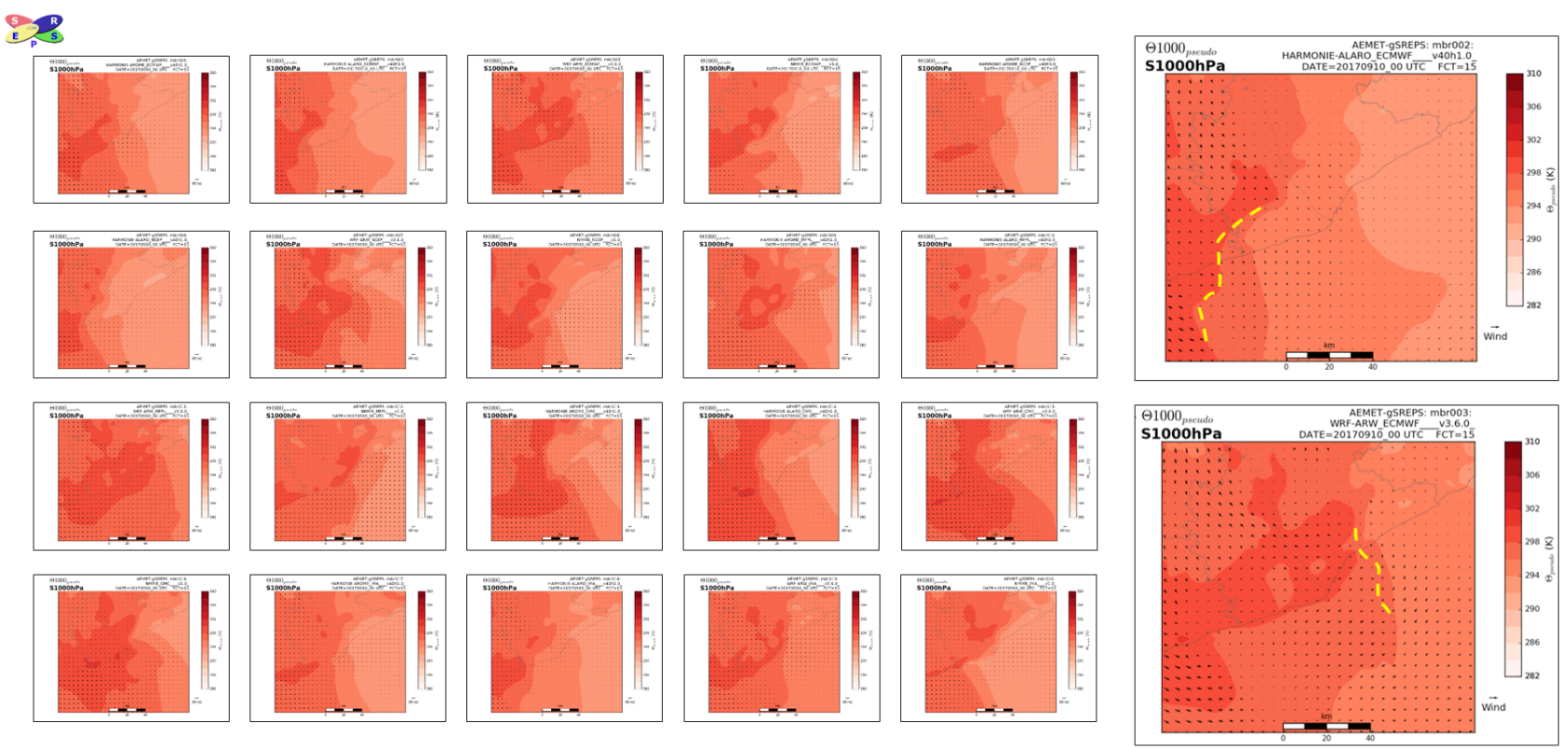

Figura 11. Salidas del campo de temperatura pseudoequivalente y viento en el nivel de $1000 \mathrm{hPa}$ del modelo probabilista AEMET- $\gamma$ SREPS para el 10-9-2017 en el alcance H+15 (a las 15 UTC). Los miembros 2 y 3 muestran gráficamente la incertidumbre asociada a la localización de la línea de convergencia.

\subsection{Predicción aeronáutica}

La tabla 1 muestra, de forma sintetizada, el METAR (observación de aeródromo) y el TAF (pronóstico de aeródromo). Se ha optado por utilizar un sencillo código de colores para facilitar el seguimiento de la secuencia temporal de la dirección del viento en superficie a lo largo del día 10-9-2017 en el aeropuerto.

En la fila superior, se muestra el viento observado que fue reportado en el METAR. La evolución a lo largo de todo el día puede seguirse de izquierda a derecha. Llama la atención la gran variabilidad: el día comenzó con un viento del S-SW, pasando posteriormente a NW que roló poco después a NE, para finalmente acabar en N-NW. A pesar de no ser un día fácil para elaborar el pronóstico del viento, los TAF emitidos desde el día anterior ya advertían de los diferentes cambios posteriormente observados. En efecto, cada discontinuidad en la dirección del viento se ubica dentro del grupo de cambio del TAF BECMG.

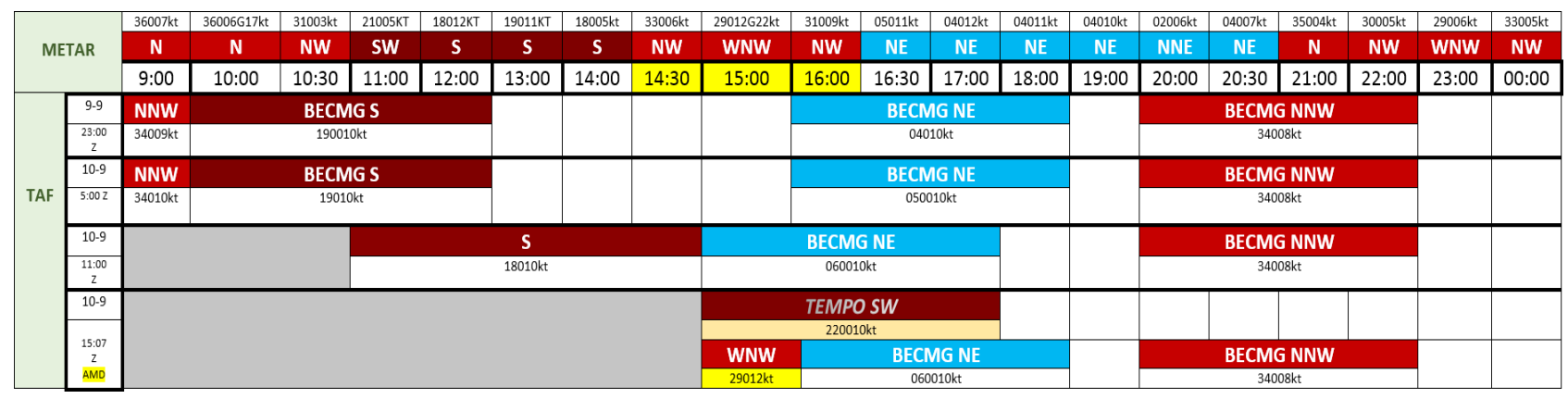

Tabla 1. METAR y TAF del viento emitidos para el 10-9-2017.

Los colores diferencian las direcciones del viento. 


\section{CONCLUSIONES}

- Una corriente de densidad mesoescalar atrapada en la costa fue la precursora del establecimiento de un entorno de cizalladura en LEBL el día 10-9-2017, entre las 16 UTC y las 18 UTC. Se trata de un fenómeno conocido, bastante estudiado en otras áreas geográficas y que recientemente se ha observado que es relativamente común en esta zona.

- El estado del arte de la predicción no permite un pronóstico estrictamente determinista de un fenómeno mesoescalar, como el que es objeto de este estudio, cuya afectación se circunscribía a un espacio muy limitado y en un reducido periodo de tiempo. Por este motivo, debe tenerse en cuenta el valor añadido que aportan los sistemas de predicción por conjuntos (EPS) mediante la consideración de diversos escenarios.

- Aun tratándose de una situación de baja predecibilidad el pronóstico de aeródromo fue muy satisfactorio, logrando predecir la mayor parte de los cambios de viento producidos ese día.

\section{AGRADECIMIENTOS}

Personal de la Oficina Meteorológica del aeropuerto de Barcelona- El Prat.

ENAIRE.

\section{REFERENCIAS}

Bengtsson, L., Andrae, U., Aspelien, T., Batrak, Y., Calvo Sánchez, F. J., de Rooy, W., Gleeson, E., Hansen Sass, B., Homileid, M., Hortal, M., Ivarsson, K.-I., Lenderink, G., Niemelä, S., Nielsen, K. P., Onvlee, J., Rontu, L., Samuelsson, P., Santos Muñoz, D., Subías Díaz-Blanco, Á., Tum, A., Toll, V., YAnG, X. y ØDEgaARd KøLgzow, M., 2017. The HARMONIE-AROME Model Configuration in the ALADIN-HIRLAM NWP System. American Meteorological Society. Monthly Weather Review, 145 (5), 1919-1935.

GonzÁlez, S., Callado, A., Werner, E., Escribà, P. y Bech, J., 2018. Coastally-Trapped Disturbances caused by the Tramontane wind on the North-Western Mediterranean: Numerical study and Sensitivity to Short-wave Radiation. Quarterly Journal of the Royal Meteorological Society. 48 pp. 
